\title{
Clinical outcomes in patients with acute myocardial infarction treated with primary percutaneous coronary intervention stratified according to duration of pain-to-balloon time and type of myocardial infarction
}

Bartłomiej Staszczak ${ }^{1}$, Zbigniew Siudak ${ }^{2}$, Krzysztof Piotr Malinowski ${ }^{3}$, Magdalena Jędrychowska ${ }^{1}$, Michał Zabojszcz ${ }^{2}$, Magdalena Dolecka-Ślusarczyk ${ }^{2}$, Agnieszka Janion-Sadowska ${ }^{2}$, Michał Susu1 ${ }^{1}$, Tomasz Tokarek ${ }^{1}$, Joanna Bartuśs Artur Pawlik ${ }^{1}$, Sylwia Socha ${ }^{1}$, Andrzej Surdacki ${ }^{1,3}$, Stanisław Bartuś ${ }^{1,3}$, Rafał Januszek $^{1,5}$

${ }^{1}$ Department of Cardiology and Cardiovascular Interventions, University Hospital, Krakow, Poland

${ }^{2}$ Collegium Medicum, Jan Kochanowski University, Kielce, Poland

${ }^{3}$ Department of Cardiology, Jagiellonian University Medical College, Krakow, Poland

${ }^{4}$ Andrzej Frycz Modrzewski Krakow University, Krakow, Poland

${ }^{5}$ University of Physical Education, Department of Clinical Rehabilitation, Krakow, Poland

\begin{abstract}
Background: Based on the clinical outcomes of patients with ST-segment elevation myocardial infarction (STEMI) and non-ST-segment elevation myocardial infarction (NSTEMI), treated with primary percutaneous coronary intervention ( $(P C I)$, this study intended to assess mortality and major adverse cardiac and cerebrovascular event (MACCE) rates according to duration of pain-to-balloon (PTB) time and type of MI. Methods: This is a retrospective cohort study based on the prospectively collected ORPKI registry which covers PCIs performed in Poland chosen between January 2014 and December 2017. Under assessment were 1,994 STEMI and 923 NSTEMI patients. Study endpoints included mortality and MACCE rates (in-hospital, 30-day, 12- and 36-month). Predictors of all-cause mortality in the overall group, STEMI and NSTEMI were assessed by multivariable analysis.

Results: Kaplan-Meier survival curve analysis did not reveal significant differences between the STEMI and NSTEMI group for all-cause mortality or MACCE at the 36-month follow-up. While in the long PTB time group, MACCE rate was significantly greater in STEMI patients when compared to NSTEMI $(p=0.004)$. Among STEMI patients, the short, medium and long PTB time groups differed significantly in the rate of all-cause mortality $(p=0.006)$ and MACCE $(p=0.04)$ at 1,095 days of follow-up, which were the greatest in the long PTB time group.

Conclusions: Before considering the length of PTB time, there were no statistically significant differences in mortality or MACCE frequency between the STEMI and NSTEMI group at 36-month follow-up. Longer PTB times are related to significantly greater mortality at the 36-month follow-up in the STEMI, but not in the NSTEMI group. (Cardiol J)

Key words: acute myocardial infarction, clinical outcomes, mortality, pain-to-balloon time, primary percutaneous coronary intervention
\end{abstract}

Address for correspondence: Rafał Januszek, MD, PhD, Department of Cardiology and Cardiovascular Interventions, University Hospital in Krakow, ul. Jakubowskiego 2, 30-688 Kraków, Poland, tel: +48 1240022 50, fax: +48 1240022 67, e-mail: jaanraf@interia.pl

Received: 3.07.2020 Accepted: 28.02.2021 Early publication date: 7.07.2021

This article is available in open access under Creative Common Attribution-Non-Commercial-No Derivatives 4.0 International (CC BY-NC-ND 4.0) license, allowing to download articles and share them with others as long as they credit the authors and the publisher, but without permission to change them in any way or use them commercially. 


\section{Introduction}

The European Society of Cardiology guidelines recommend primary percutaneous coronary intervention (pPCI) as the preferred treatment for patients with acute myocardial infarction (AMI). In patients with ST-segment elevation myocardial infarction (STEMI), it is advocated that pPCI be performed $\leq 2 \mathrm{~h}$ from diagnosis, and at no longer than $24 \mathrm{~h}$ from diagnosis [1]. While the time of invasive treatment in patients with non-ST-segment elevation myocardial infarction (NSTEMI) is less restrictive and mainly based on risk stratification. It depends on the initial risk stratification, and very high-risk patients should be treated with pPCI less than $2 \mathrm{~h}$ from diagnosis. For high-risk patients, this time may be prolonged to $24 \mathrm{~h}$, while for intermediate-risk patients, an invasive strategy could be introduced even up to $72 \mathrm{~h}$ [2]. In the current study, based on the local and distant clinical outcomes of patients with AMI treated with pPCI, the intention was to assess and compare clinical outcomes according to the duration of pain-to-balloon (PTB) time, and its relationship with mortality, major adverse cardiac and cerebrovascular events (MACCE) in light of myocardial infarction type (STEMI vs. NSTEMI). The purpose of this was to evaluate potential factors that may have an effect on treatment results. Another important issue is the impact of selected factors on major endpoints such as mortality, depending on the follow-up period and type of AMI based on the analysis of a selected local population.

Therefore, the goal of the current study was to examine the relationship between clinical outcomes and the duration of PTB time in STEMI and NSTEMI patients, as well as predictors of mortality.

\section{Methods}

\section{Study design, population setting and eligibility criteria}

This is a retrospective cohort study based on the prospectively collected ORPKI registry, which covers almost all PCI procedures performed in Poland. This registry was described in previously published studies [3]. Patients were chosen between January 2014 and December 2017. All consecutive patients treated with PCI and with diagnosed AMI according to the current European guidelines were included in the present study $[1,2]$. Then, data received from the ORPKI registry were matched with the data received from the National
Health Fund. The procedure for merging data and its characteristics were described in a previously published work [4].

\section{Study definitions}

Pain-to-balloon time was defined as the period from the occurrence of the earliest AMI symptoms to the first inflation of a catheter balloon within the culprit lesion. For the purpose of the current study, all of the included patients in the analysis were divided according to length of PTB time: patients with shorter PTB time than $3 \mathrm{~h}$ (short PTB time group), with the PTB time longer than $3 \mathrm{~h}$ but shorter than $12 \mathrm{~h}$ (medium PTB time group) and with the PTB time longer than $12 \mathrm{~h}$ but shorter than $24 \mathrm{~h}$ (long PTB time group). The overall group of AMI patients was also analysed according to the type of AMI: STEMI and NSTEMI. First contact-to-balloon time was defined as the duration from first medical contact of the patient with AMI to catheter balloon inflation in the culprit artery.

\section{Primary PCI protocol}

The selection of interventional strategy and device usage were left to the discretion of the attending physician. Pharmacological treatment was ordered according to routinely recommended protocols $[1,2]$.

\section{Study endpoints}

All-cause mortality rate was distinguished among the primary study endpoints, which were assessed at selected time points: in-hospital, 30-days, 12- and 36-months for death rates while 30-days, 12- and 36-months were for MACCE rates.

\section{Statistical analysis}

Categorical variables are presented as numbers and percentages. Continuous variables are expressed as means (standard deviation [SD]) or median (interquartile range [IQR]), where applicable. Normality was assessed using the Shapiro-Wilk test. Equality of variance was assessed using the Levene's test. Differences between the two groups were compared using the Student or Welch t-test depending on the equality of variances for normally distributed variables. The Mann-Whitney U test was used for non-normally distributed continuous variables. Categorical variables were compared via the Pearson $\chi^{2}$ test or Fisher exact test if $20 \%$ of cells had an expected count of less than 5 (the Monte Carlo simulation for the Fisher test used tables of higher dimensions than $2 \times 2$ ). Multiple group comparisons were performed using analysis 
of variance (ANOVA) or the Kruskal-Wallis test. The Tukey-Kramer HSD test or the Steel-Dwass method was used for post-hoc comparisons. In the case of categorical parameters and survival analyses, the Benjamini-Hochberg procedure was used to adjust the p-value. Univariable and multivariable Cox proportional hazard models were applied to identify predictors of MACCE and death. The set of factors in the adjusted model included: time of day, type of MI, age, smoking status, hypertension, Killip class grade, gender, diabetes, kidney disease, prior stroke, MI and/or PCI, cardiac arrest at baseline, chronic obstructive pulmonary disease, treatment with acetylsalicylic acid at baseline as well as Thrombolysis in Myocardial Infarction (TIMI) flow grade before and after PCI. All statistical analyses were performed with $\mathrm{JMP}^{\circledR}$, Version 14.2.0 (SAS Institute INC., Cary, NC, USA). All statistical tests were two-sided, the level of $p<0.05$ was considered statistically significant.

\section{Results}

\section{Study population}

The present study included 1,994 patients with STEMI and 923 patients with NSTEMI, thus a total of 2,917 consecutive patients admitted to the hospital with AMI and treated with pPCI. All patients were divided into three groups according to the duration of PTB time, as described above. In addition, patients were analysed depending on the type of infarction in the STEMI and NSTEMI patient groups. When considering the STEMI group and PTB times, there where $900(45.1 \%)$ patients in the first, 949 (47.6\%) patients in the second and 145 $(7.3 \%)$ patients in the third group. In the NSTEMI group, there were $100(10.8 \%)$ in the first group, 502 $(54.4 \%)$ in the second and $321(34.8 \%)$ in the third one. When considering the overall group of patients with AMI, the short, medium and long PTB time groups differed significantly in the rate of all-cause mortality $(\mathrm{p}=0.04)$, but not MACCE $(\mathrm{p}=0.88)$ at 1,095 days of the follow-up period (Fig. 1A, B). The mortality rate was greatest in the long PTB time group compared to those with medium and short PTB times (Fig. 1A). Significant predictors of allcause mortality at selected time points (in-hospital, 30-day, 12 -month and 36-month), assessed by multivariable analysis, are presented in Figure 2A-D.

\section{STEMI vs. NSTEMI}

General characteristics. When comparing mean age, patients in NSTEMI group were significantly older than in the STEMI group (67.3 \pm \pm 12.2 vs. $65.3 \pm 12.1, \mathrm{p}<0.05)$. Patients in the NSTEMI group suffered more often from kidney failure ( $4 \%$ vs. $2.2 \%, \mathrm{p}<0.05)$ and arterial hypertension $(67.6 \%$ vs. $61.5 \%, \mathrm{p}<0.05)$. These and other indices are presented in Table 1.

Procedural indices. Patients from the STEMI group were significantly more frequently treated from femoral access and less from radial in comparison to the NSTEMI group ( $\mathrm{p}<0.05)$. These and other procedural indices are presented in Table 2.

Study endpoints. In-hospital mortality $(7 \%$ vs. $3.6 \%, \mathrm{p}<0.05)$ and 30 -day mortality $(7.7 \%$ vs. $5.1 \%, \mathrm{p}<0.05)$ were significantly greater among STEMI patients compared to NSTEMI, and at the following time points (12-month and 36-month mortality), they did not differ significantly (Table 3 ). Kaplan-Meier survival curve analysis did not reveal significant differences between the STEMI and NSTEMI groups in all-cause mortality $(\mathrm{p}=0.64)$ or MACCE ( $\mathrm{p}=0.17)$ at 36 months of follow-up.

Pain-to-balloon times. When considering the short PTB time group, Kaplan-Meier survival curves analysis did not reveal significant differences between the STEMI and NSTEMI groups at 1,095 days of follow-up in all-cause mortality $(\mathrm{p}=0.62)$ or MACCE $(\mathrm{p}=0.28)$ rates (Fig. $3 \mathrm{~A}, \mathrm{~B})$. Similarly, in the medium PTB time group, Kaplan-Meier survival curves analysis did not show significant differences between the STEMI and NSTEMI groups at 1,095 days of follow-up in allcause mortality $(\mathrm{p}=0.14)$ or MACCE $(\mathrm{p}=0.86)$ rates (Fig. $3 \mathrm{C}, \mathrm{D}$ ). While in the long PTB time group, Kaplan-Meier survival curve analysis did not demonstrate significant differences at 1095 days of follow-up in all-cause mortality $(\mathrm{p}=0.18)$, however, it was significantly greater in STEMI patients when compared to NSTEMI for MACCE $(\mathrm{p}=0.004)$ rates (Fig. $3 \mathrm{E}, \mathrm{F})$.

\section{Pain-to-balloon time in STEMI}

General characteristics. When considering PTB times, patients from the long PTB time group were significantly older $(\mathrm{p}<0.001)$ and were less often males $(p=0.006)$, smokers $(p=0.01)$, while they were found to have an incidence of prior stroke $(\mathrm{p}=0.04)$ and MI $(\mathrm{p}=0.02)$ more often. These and other indices are presented in Table 1.

Procedural indices. Patients from the short PTB time group presented single-vessel disease (SVD) significantly more often, while multi-vessel disease (MVD) was less frequent in comparison to patients from the medium and long PTB time groups $(\mathrm{p}<0.001)$. These and other procedural indices are presented in Table 2. 


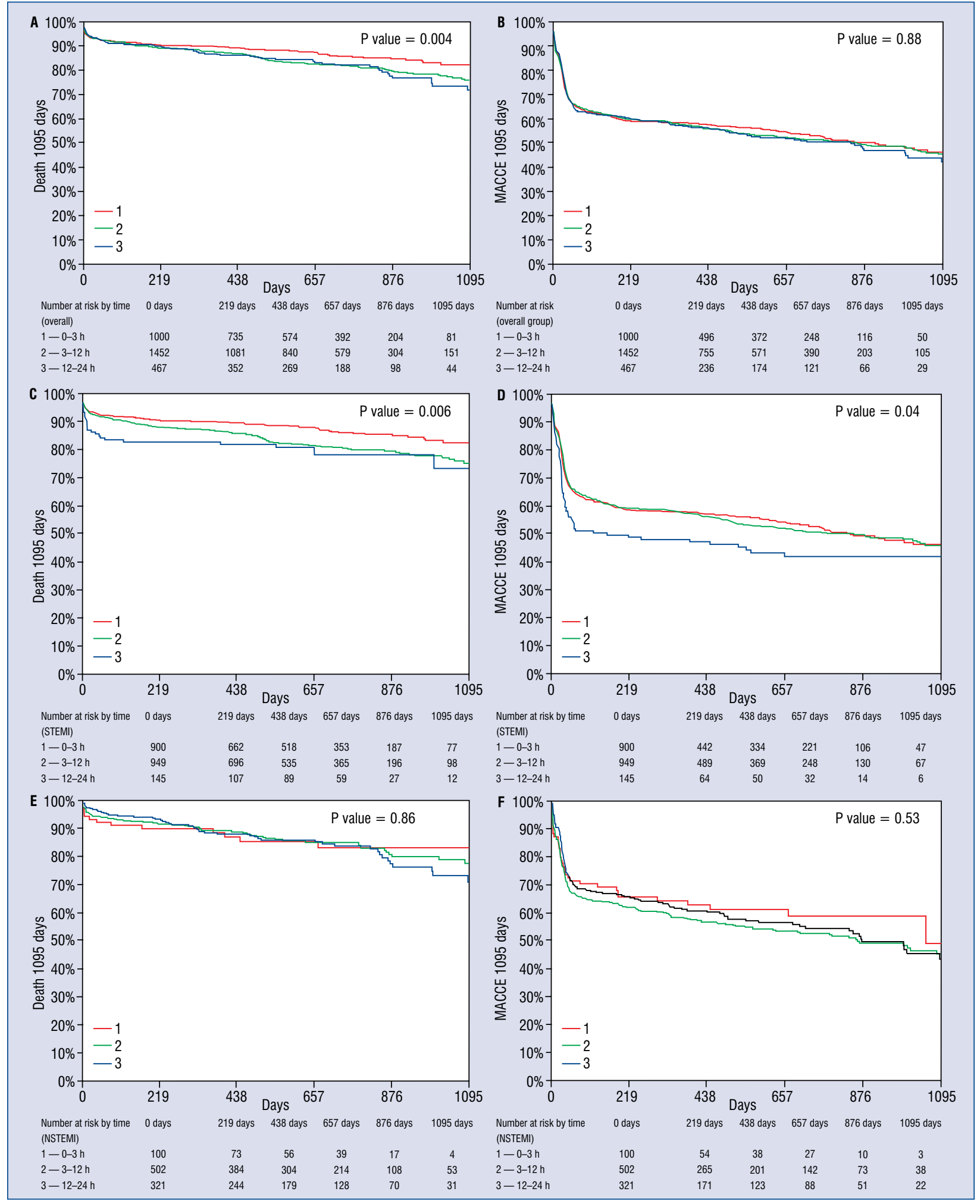

Figure 1. Selected study endpoints presented as Kaplan-Meier survival curves compared in the ST-segment elevation myocardial infarction (STEMI) and non-ST-segment elevation myocardial infarction (NSTEMI) groups according to the duration of pain-to-balloon time; A. All-cause mortality for 1,095 days of follow-up in patients with pain-to-balloon time shorter than $3 \mathrm{~h}$; B. Major adverse cardiac and cerebrovascular events (MACCE) incidence for 1,095 days of follow-up in patients with pain-to-balloon time shorter than $3 \mathrm{~h}$; C. All-cause mortality for 1,095 days of follow-up in patients with pain-to-balloon time longer than $3 \mathrm{~h}$ but shorter than $12 \mathrm{~h}$; D. MACCE incidence for 1,095 days of followup in patients with pain-to-balloon time longer than $3 \mathrm{~h}$ but shorter than $12 \mathrm{~h}$; E. All-cause mortality for 1,095 days of follow-up in patients with pain-to-balloon time longer than $12 \mathrm{~h}$ but shorter than $24 \mathrm{~h}$; F. MACCE incidence for 1,095 days of follow-up in patients with pain-to-balloon time longer than $12 \mathrm{~h}$ but shorter than $24 \mathrm{~h}$. 


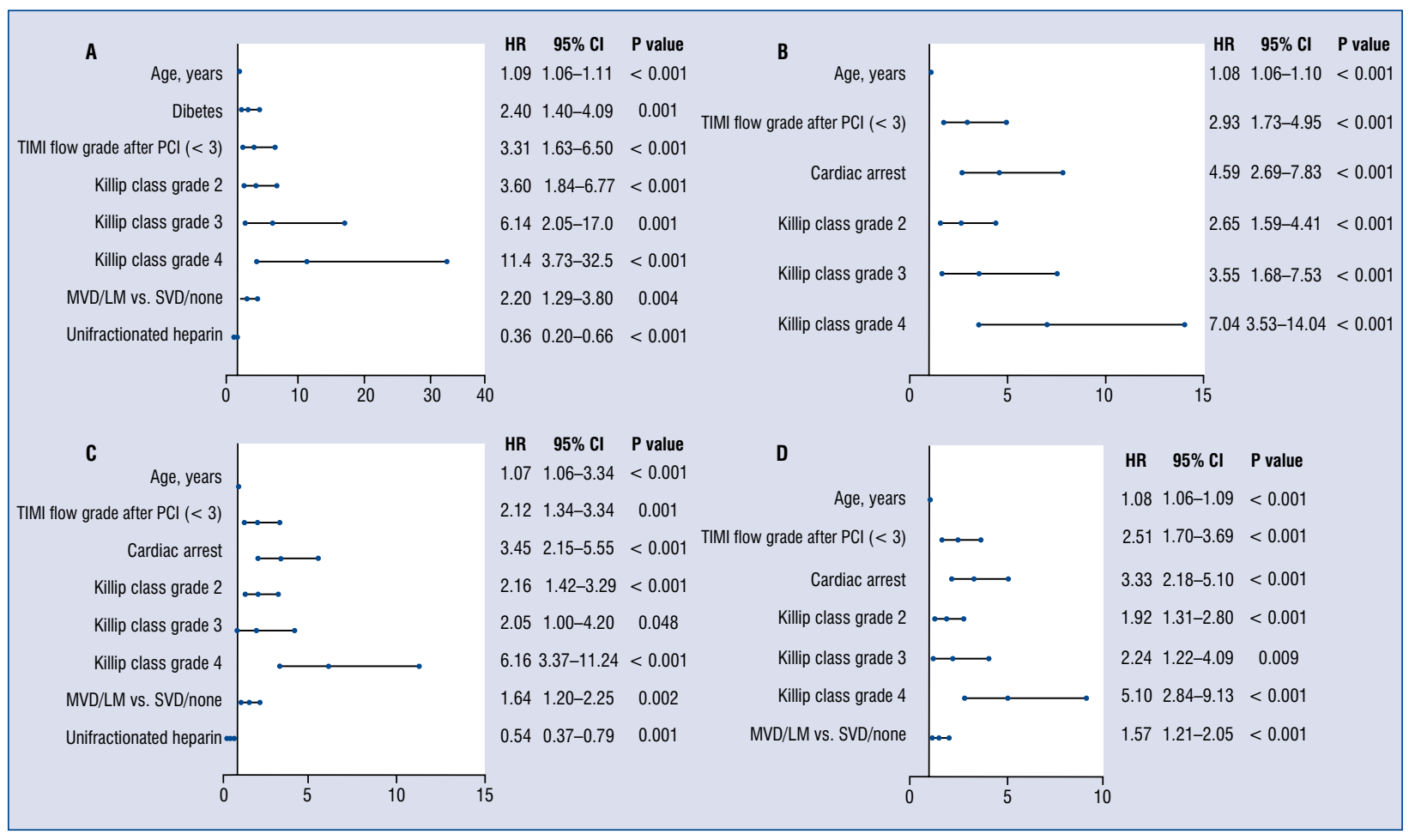

Figure 2. Predictors of all-cause mortality assessed by multivariable analysis in the overall group of patients - acute myocardial infarction; A. In-hospital; B. 30-day; C. 12-month; D. 36-month; Cl - confidence interval; HR - hazard ratio; LM — left main coronary artery; MVD — multi-vessel disease; SVD — single-vessel disease; $\mathrm{PCI}$ - percutaneous coronary intervention; TIMI — Thrombolysis in Myocardial Infarction.

Study endpoints. The 30-day $(p=0.025)$, 12 -month $(\mathrm{p}=0.019)$ and 36-month $(\mathrm{p}=0.003)$ mortality rates were greatest among the long PTB time group compared to the medium and short PTB time groups (Table 3$)$. The 30 -day $(\mathrm{p}=0.001)$ MACCE frequency was significantly higher in the long PTB time group in comparison to others (Table 3). When considering the STEMI group of patients, the short, medium and long PTB time groups significantly differed in the rate of all-cause mortality $(\mathrm{p}=0.006)$ and MACCE $(\mathrm{p}=0.04)$ at 1,095 days of the follow-up period, which was the greatest in the long PTB time group compared to the medium and short ones (Fig. 1A, B). Significant predictors of all-cause mortality at selected time points (in-hospital, 30-day, 12-month and 36 -month) assessed by multivariable analysis are presented in Figure 4A-D.

\section{Pain-to-balloon time in NSTEMI}

General characteristics. General characteristics are presented in Table 1.

Procedural indices. Radial access was significantly more frequently used in the long PTB time group when compared to others, while femoral access was implemented less often $(\mathrm{p}<0.001)$. Patients with SVD occurred significantly more frequently in the short PTB time group, while MVD patients were most present in the long PTB time group $(\mathrm{p}=0.03)$. These and other procedural indices are presented in Table 2.

Study endpoints. There were no significant differences in all-cause mortality or MACCE rates between all of the three assessed groups at all follow-up time points (Table 3). Using Kaplan-Meier survival curves analysis, it was confirmed that there is no significant difference in the frequency of all-cause mortality $(\mathrm{p}=0.86)$ and MACCE $(p=0.53)$ rates between short, medium and long PTB time groups (Fig. 3E, F). Significant predictors of all-cause mortality at selected time points (in-hospital, 30-day, 12-month and 36-month) assessed by multivariable analysis are presented in Figure 5A-D.

\section{Predictors of all-cause mortality}

Overall group. Among significant positive predictors of all-cause in-hospital mortality it was found that age, diabetes, TIMI flow grade after PCI, Killip class grade before PCI, MVD with or without 


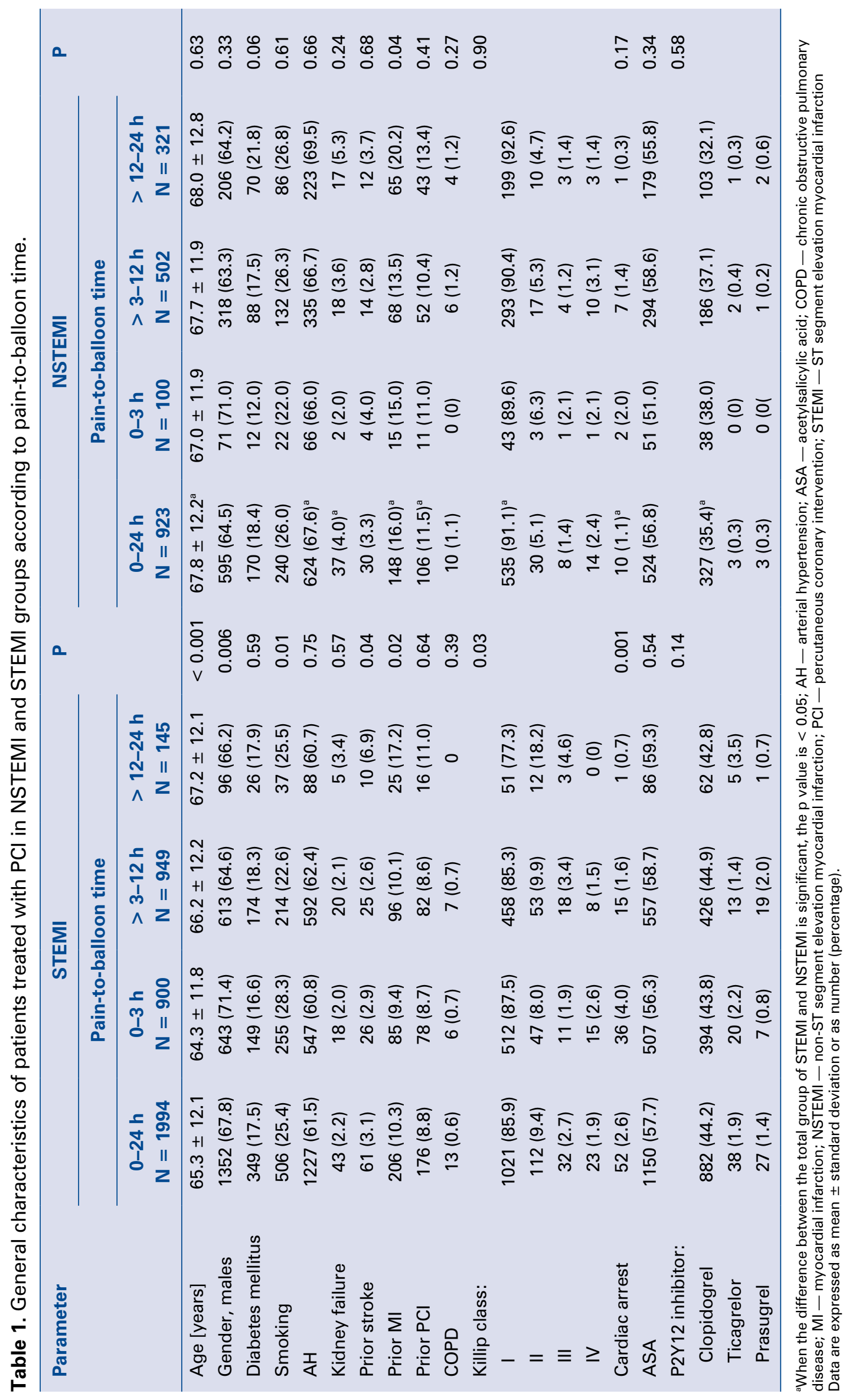




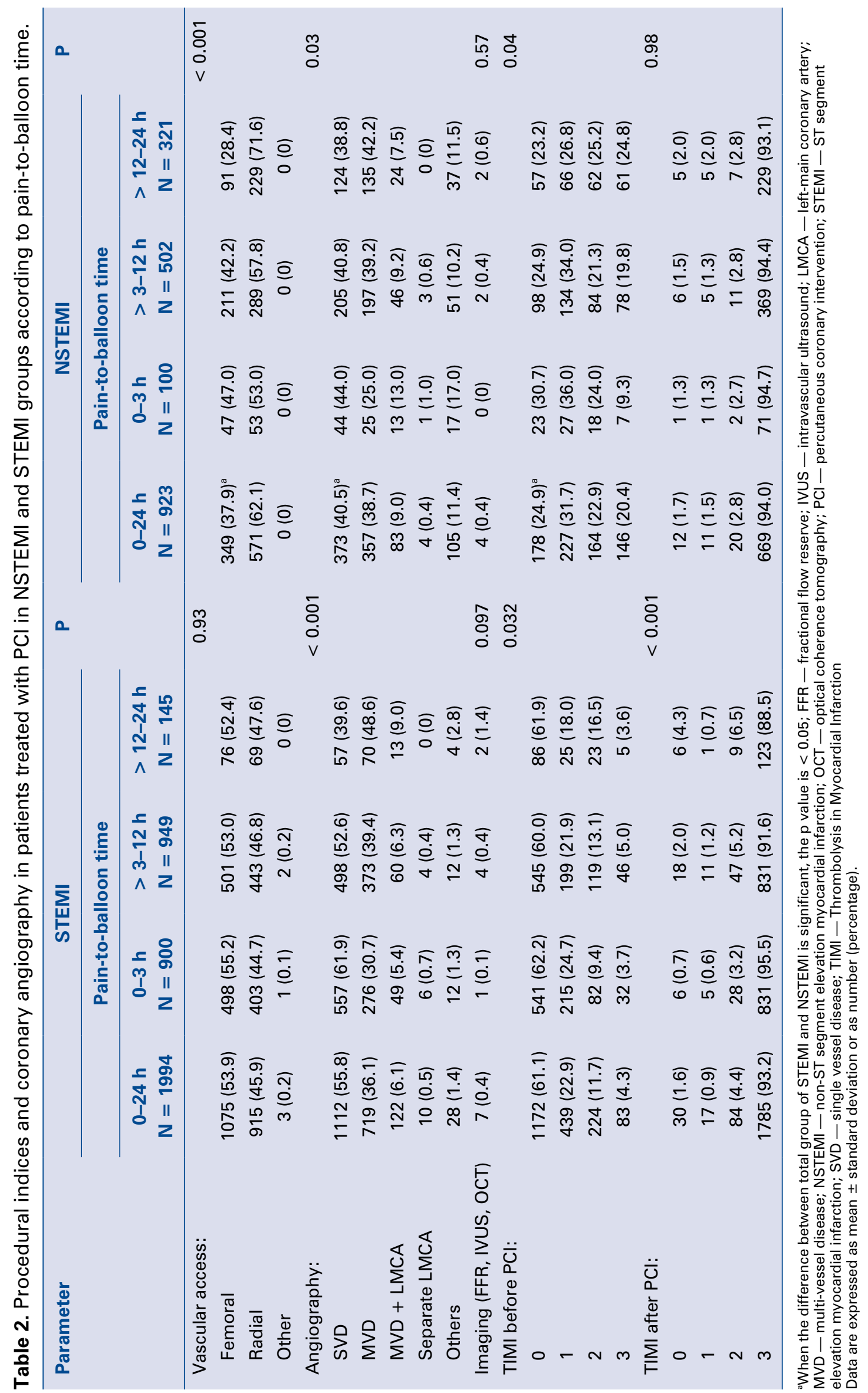




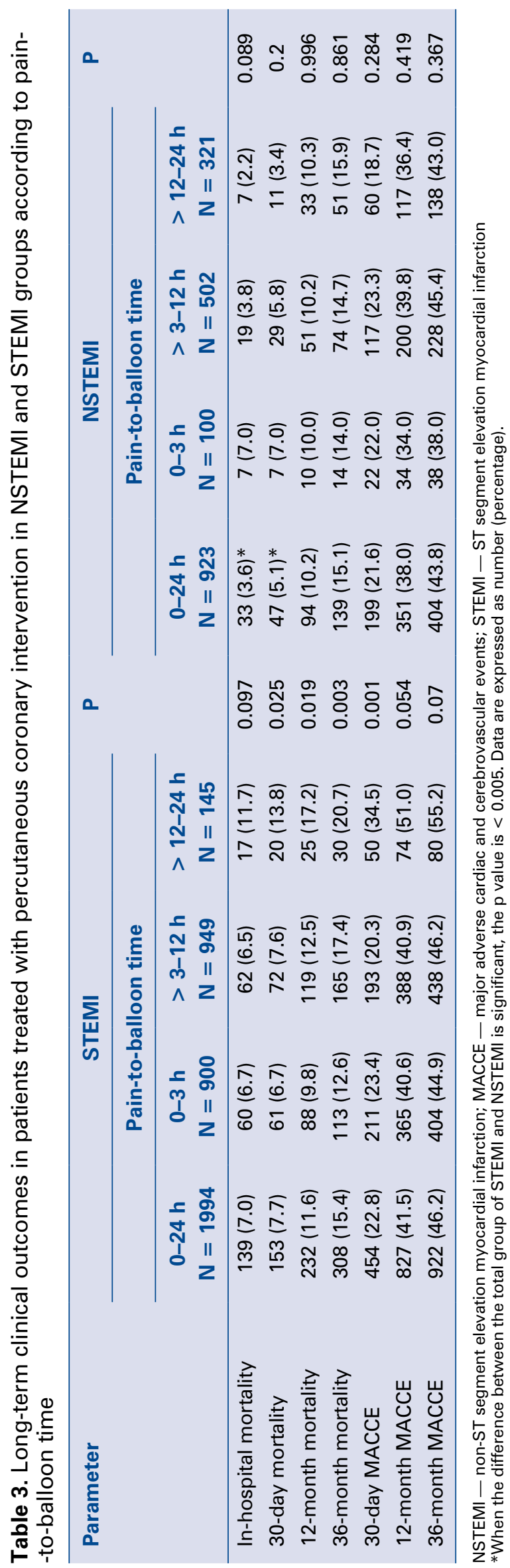

left main coronary artery (LMCA) involvement, while among the negative predictors it was treatment with unfractionated heparin (Fig. 2A). At 30-day follow-up, among positive predictors of all-cause mortality it was confirmed that age, TIMI grade flow after PCI, cardiac arrest and Killip class grade before PCI (Fig. 2B). At 12-month follow-up, age, TIMI flow grade after PCI, cardiac arrest, Killip class grade before PCI, MVD \pm \pm LMCA involvement were found to be significant predictors of all-cause mortality, while treatment with unfractionated heparin was a negative predictor (Fig. 2C). Among significant positive predictors of all-cause mortality at 36-month follow-up there were age, TIMI flow grade after PCI, cardiac arrest, Killip class grade before PCI and MVD \pm LMCA involvement (Fig. 2D).

STEMI. Confirmed herein, was age, cardiac arrest, Killip class grade before PCI and MVD \pm \pm LMCA involvement being among significant positive predictors of all-cause in-hospital mortality, while treatment with unfractionated heparin was among negative predictors (Fig. 4A). Among significant positive predictors of all-cause mortality found at 30-day follow-up was age, cardiac arrest and Killip-class grade (Fig. 4B). While at 12-month follow-up, age, TIMI flow grade after PCI, cardiac arrest and Killip class grade were confirmed as significant positive predictors of all-cause mortality, and treatment with unfractionated heparin was found to be negative predictor (Fig. 4C). Among significant positive predictors of all-cause mortality at 36-month follow-up age, TIMI flow grade after PCI, cardiac arrest and Killip class grade before PCI were confirmed (Fig. 4D).

NSTEMI. Among significant positive predictors of in-hospital all-cause mortality confirmed age, TIMI flow grade after PCI and Killip class grade before PCI were confirmed (Fig. 5A). At 30-day follow-up, age, cardiac arrest and TIMI flow grade after PCI as positive predictors of all-cause mortality were confirmed, while among negative predictors was the smoking habit Age, cardiac arrest and MVD \pm LMCA involvement were found to be significant predictors of all-cause mortality at 12-month follow-up (Fig. 5C), while at 36-month: age, TIMI flow grade after PCI, cardiac arrest and MVD \pm LMCA involvement (Fig. 5D). Among negative predictors of all-cause mortality at 36-month follow-up smoking habit was found (Fig. 5D).

\section{Discussion}

This study demonstrated that in the overall group of patients there were no significant differences in all-cause mortality between the STEMI 


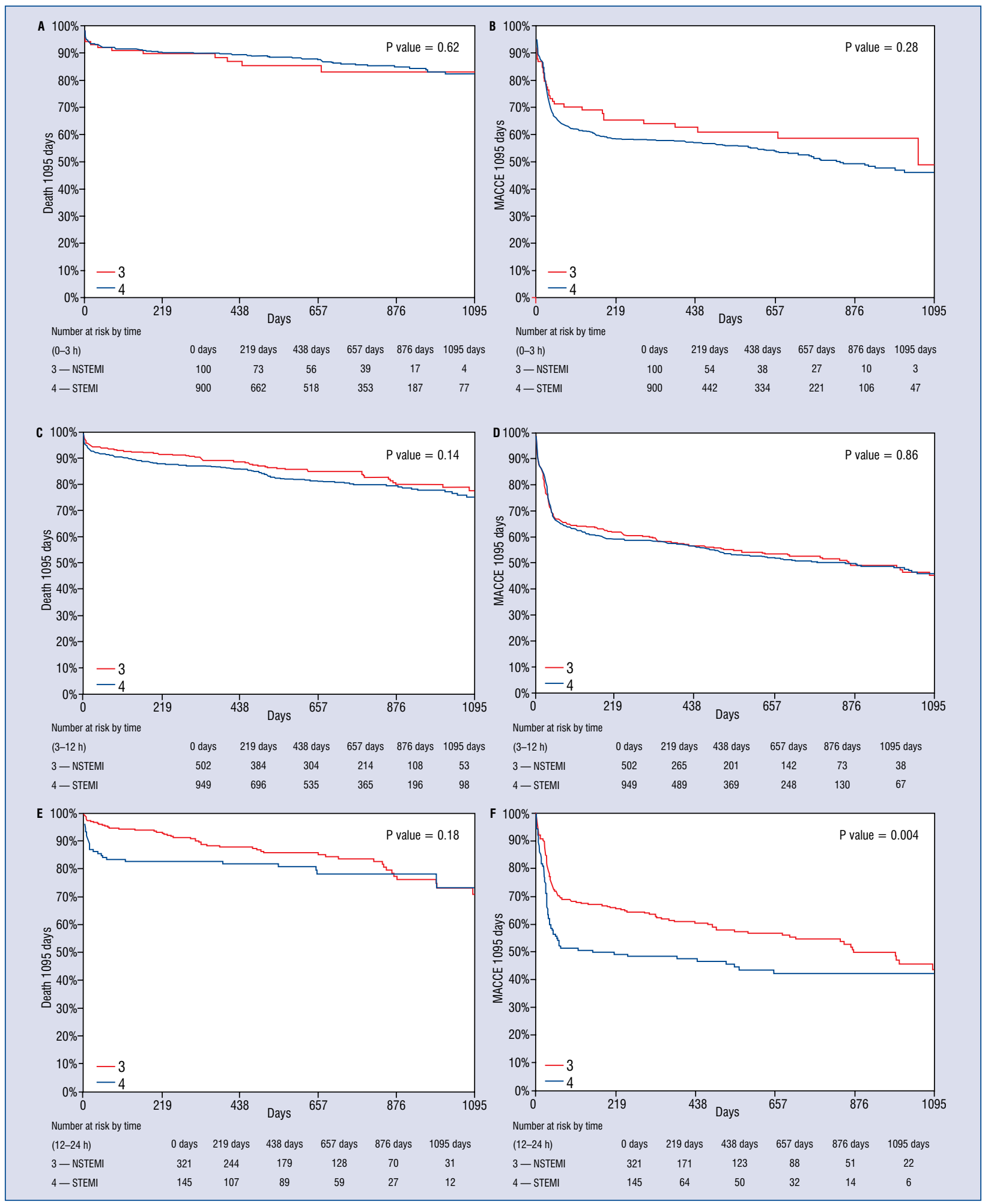

Figure 3. Selected study endpoints presented as Kaplan-Meier survival curves compared in short, medium and long pain-to-balloon time groups according to the type of myocardial infarction (acute myocardial infarction [AMI] - overall group, ST-segment elevation myocardial infarction [STEMI] and non-ST-segment elevation myocardial infarction [NSTEMI]); A. All-cause mortality for 1,095 days of follow-up in the AMI group; B. Major adverse cardiac and cerebrovascular events (MACCE) incidence for 1,095 days of follow-up in the AMI group; C. All-cause mortality for 1,095 days of follow-up in the STEMI group; D. MACCE incidence for 1,095 days of follow-up in the STEMI group; E. All-cause mortality for 1,095 days of follow-up in the NSTEMI group; F. MACCE incidence for 1,095 days of followup in the NSTEMI group. 


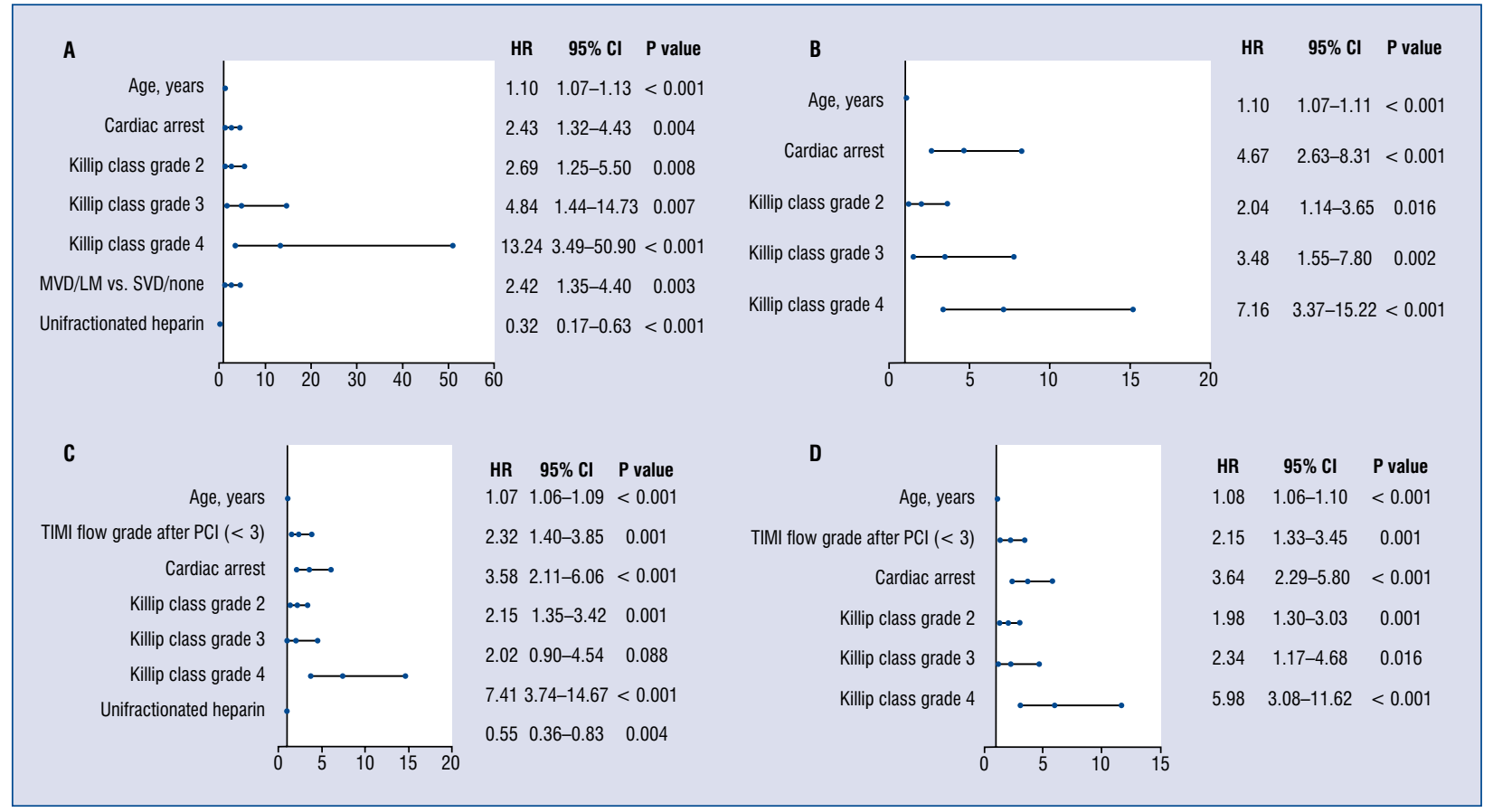

Figure 4. Predictors of all-cause mortality assessed by multivariable analysis in the ST-segment elevation myocardial infarction (STEMI) group; A. In-hospital; B. 30-day; C. 12-month; D. 36-month. Cl - confidence interval; HR - hazard ratio; LM — left main coronary artery; MVD — multi-vessel disease; SVD — single-vessel disease; $\mathrm{PCI}$ - percutaneous coronary intervention; TIMI — Thrombolysis in Myocardial Infarction.

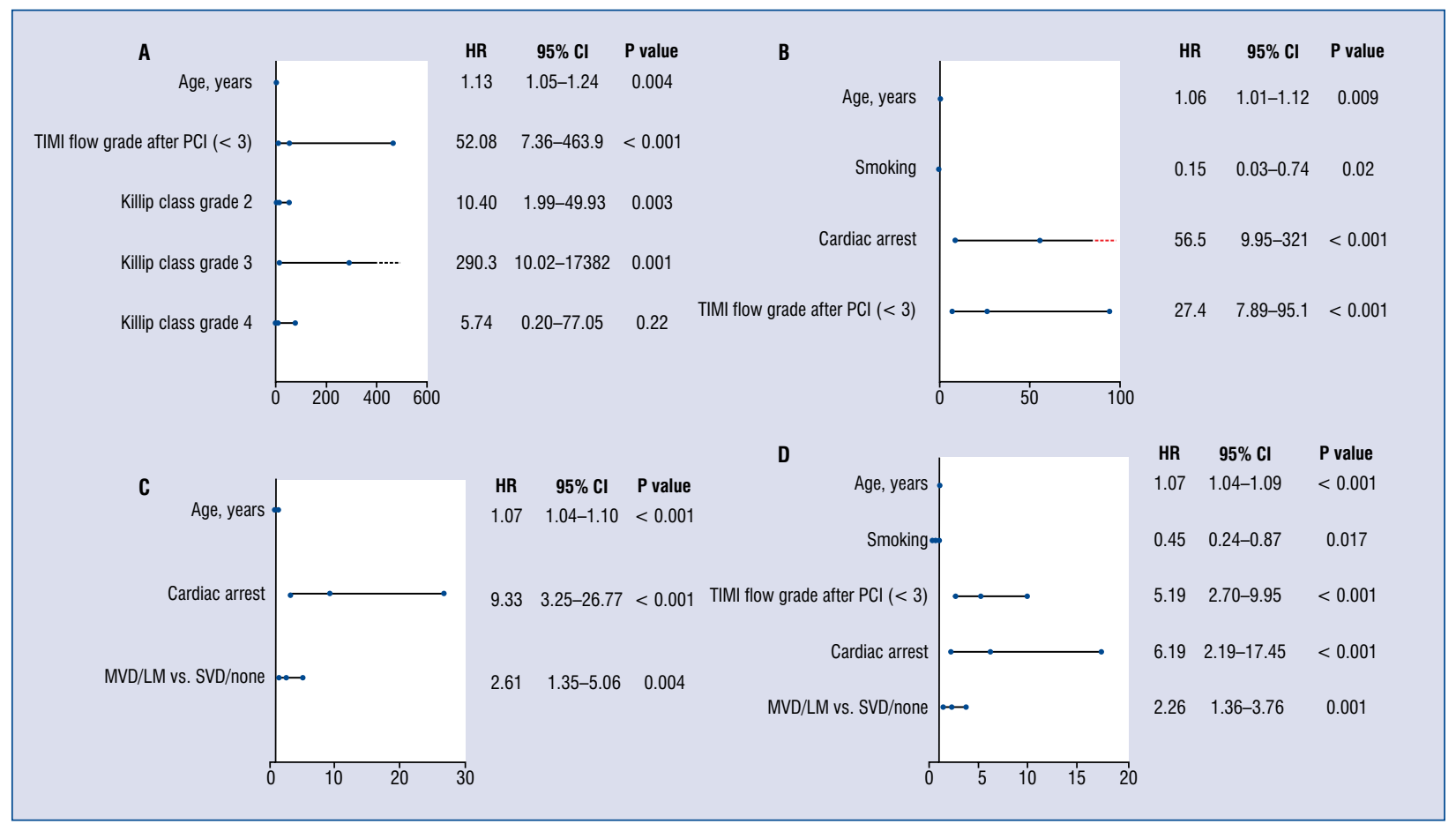

Figure 5. Predictors of all-cause mortality assessed by multivariable analysis in the non-ST-segment elevation myocardial infarction (NSTEMI) group; A. In-hospital; B. 30-day; C. 12-month; D. 36-month. Cl — confidence interval; HR - hazard ratio; LM - left main coronary artery; MVD - multi-vessel disease; SVD — single-vessel disease; $\mathrm{PCl}$ - percutaneous coronary intervention; TIMI — Thrombolysis in Myocardial Infarction. 
and NSTEMI groups at the 36-month follow-up. While there were no statistical differences between these groups when comparing the short and medium PTB time groups, the MACCE rate, but not mortality, was significantly greater in the STEMI group when compared to NSTEMI for the long PTB time group at the 36-month follow-up. Mortality rate at 36 months was significantly greater in the long PTB time group compared to the others in the overall AMI and STEMI groups, nevertheless, it remained non-significant for NSTEMI. MACCE rate was also significantly greater in the long PTB time group compared to others in STEMI patients, but not in the NSTEMI and overall AMI groups at 36 -month follow-up. There were no clear differences between individual types of MIs in terms of mortality predictors, although the results indicate that the severity of coronary artery atherosclerosis (MVD/LMCA vs. SVD/none) was significant for the NSTEMI and overall AMI groups, but not for STEMI patients. Other predisposing factors for increased mortality were similar irrespective of the type of infarction or follow-up, and included age, clinical image at admission as well as arterial patency before and after surgery.

It is well established that the shorter the duration of ischemia, the smaller the myocardial damage and better long-term prognosis and survival. There are many different time points in the guidelines proposed by the European Society of Cardiology. The door-to-balloon (DTB) time is a well-established predictor of survival $[1,2]$. All of the available measures are taken to shorten the DTB time and it seems that the field in which we still can make progress to reduce ischemia is PTB time. Comparing DTB time to the onset of symptoms, and further to balloon time, Park et al. [5] revealed that the median PTB time was $3.2 \mathrm{~h}$ and it was associated with increased 1-year mortality. In our study, 1-year mortality was remarkably higher in the STEMI and long PTB time groups. Evaluating symptom to balloon time, Alsamara et al. [6] noted that its longer duration was associated with worse left ventricular ejection fraction (LVEF), which could be responsible for worse future clinical outcomes. In the present study, longer PTB time was related to higher 30-day mortality in the STEMI group, and inversely, mortality experienced a decrease along with the longer delay in PTB time in the NSTEMI group. Considering long-term mortality, longer PTB time was associated with worse outcomes among the STEMI group, no such significant differences were noted among NSTEMI patients. Chen et al. [7] verified that DTB time $<60 \mathrm{~min}$ is associated with better blood flow assessed by the TIMI flow grade scale in the infarct-related artery and lower 30-day mortality rates. In the current work, TIMI flow grade 3 after the procedure was an independent predictor of long- and short-term mortality in all groups of patients, and it was also related with shorter PTB time. Halkin et al. [8] have created the CADILLAC score for predicting mortality after PCI for patients with AMI. Among featured parameters, we may find: Killip class $2-3$, final TIMI flow of $0-2$, age $>65$ years, three-vessel disease, anemia, renal insufficiency and baseline LVEF $<40 \%$. Another study comparing short- and long-term outcomes for patients after PCI due to MI showed that the STEMI group was associated with a higher risk of short-term mortality, while NSTEMI was associated with a higher risk of long-term mortality [9]. Results herein, confirmed higher in-hospital and 30-day mortality rate among the STEMI group, but what is of more importance, it was indicated that the highest mortality rate was among patients with the longest PTB time $>12-24 \mathrm{~h}$. De Luca et al. [10] created the Zwolle Risk Score for STEMI patients, which includes 6 independent predictors of 30-day mortality among patients with STEMI and allows estimating short-term mortality risk. In the present study, 5 of them were confirmedas significant predictors of in-hospital and 30-day mortality. Focusing on the STEMI group with the shortest PTB time of $<3 \mathrm{~h}$ and having the best short-term outcomes, it was noted that a majority of patients had SVD, were in the Killip class 1 and had TIMI flow 3 after PCI, consequently, finding themselves at lower risk according to the Zwolle Risk Score [10]. In further analysis, TIMI flow after PCI $<3$ was a significant predictor of mortality in almost all groups of patients and time points of follow-up, which is consistent with the results obtained in the study by Karwowski et al. [11] who noted that mortality rates in patients with final TIMI $0-1$ and 2 scores were not significantly different, and only achieving final TIMI 3 in the infarct-related artery improves outcomes in NSTEMI patients. LVEF is one of the most commonly noted in-hospital and 1-year mortality predictors, however, we were not able to assess it due to a lack of this data in the analyzed database [12, 13]. Killip class scale remains in close correlation with LVEF. In the present study, Killip class 2-4 tended to be a strong positive predictor of adverse outcomes in the STEMI as well as NSTEMI groups. Postprocedural TIMI flow $>3$ as a negative predictor of death is mentioned by De Luca et al. [10] and 
Karwowski et al. [11]. Garadah et al. [14] indicated higher in-hospital mortality among STEMI compared to NSTEMI patients, which corresponds with the present results. Interestingly, in the current observation, in-hospital mortality rate increased with longer PTB time in the STEMI group while it decreased in the NSTEMI group. This may suggest that proper preparation of patients admitted with NSTEMI could prevent post-PCI complications such as pulmonary edema, aggravation of coexisting anemia or contrast induced nephropathy. Inversely, in the STEMI group, prolongation of PTB time is responsible for the worst outcomes. In a 1-year observation, Pocock et al. [13] noted higher overall mortality rate among NSTEMI patients compared to the STEMI group. Analogous results were obtained by Chan et al. [9], but noting that patients in the NSTEMI group were older and had a greater burden of comorbidities. In the present analysis, after 1 year of follow-up, the difference in mortality rate among the STEMI and NSTEMI groups slowly decreased (11.6\% vs. $10.2 \%)$ and the risk of death at 3-year follow-up was practically equal in both groups (15.4 vs. 15.1).

In 1 paper published to date, a benefit in 1-year follow-up in terms of mortality and myocardial reinfarction of complete revascularization in patients with MVD and STEMI, as well as NSTEMI over the incomplete revascularization was demonstrated [15]. However, in the present study, the number of patients who underwent incomplete revascularization was very small due to the fact that all patients, except for a few cases, had concurrent or staged complete revascularization of significant stenosis, which were confirmed by angiography or additional imaging (intravascular ultrasound) and functional evaluation (fractional flow reserve) of the coronary circulation. Patients who did not undergo complete revascularization, despite the presence of significant stenosis, were few and belonged to the group of very high-risk patients, e.g. palliative patients, with many internal diseases, chronically immobilized (patients after a stroke), planned to undergo surgical treatment or were at high risk of bleeding.

\section{Limitations of the study}

The main limitations of the study are the lack of a range of data that could have had significant impact on the results of calculations, which was conditioned by the nature of the registry being analyzed. The missing data firstly included LVEF, biochemical indicators such as creatinine or myo- cardial damage indicators, as well as data on the type of stents and techniques used during $\mathrm{pPCI}$.

\section{Conclusions}

Before considering the duration of PTB time, no statistically significant differences were observed in mortality or MACCE frequency between the STEMI and NSTEMI groups at 36-month follow-up. Mortality rate at 36 months was significantly greater in the long PTB time group compared to the other groups in the overall AMI group and STEMI group, while it remained non-significant for NSTEMI. The MACCE rate was also significantly greater in the long PTB time group compared to others in STEMI patients, but not in the NSTEMI and overall AMI groups during 36-month follow-up. Based on the available data in the current analysis, there are no clear differences between individual types of MIs in terms of mortality predictors, although the results indicate that the severity and extent of atherosclerosis dissemination in the coronary arteries was significant for the NSTEMI and overall AMI groups, but not for STEMI patients. Other predisposing factors of increased mortality were similar irrespective of the type of infarction and follow-up, and included age, clinical image at admission, as well as arterial patency before and after surgery.

\section{Conflict of interest: None declared}

\section{References}

1. Ibanez B, James S, Agewall S, et al. 2017 ESC Guidelines for the management of acute myocardial infarction in patients presenting with ST-segment elevation: The Task Force for the management of acute myocardial infarction in patients presenting with ST-segment elevation of the European Society of Cardiology (ESC). Eur Heart J. 2018; 39: 119-177.

2. Roffi M, Patrono C, Collet JP, et al. 2015 ESC Guidelines for the management of acute coronary syndromes in patients presenting without persistent ST-segment elevation. Eur Heart J. 2015; 37(3): 267-315, doi: 10.1093/eurheartj/ehv320.

3. Januszek RA, Dziewierz A, Siudak Z, et al. Diabetes and periprocedural outcomes in patients treated with rotablation during percutaneous coronary interventions. Cardiol J. 2018 [Epub ahead of print], doi: 10.5603/CJ.a2018.0102, indexed in Pubmed: 30234901.

4. Januszek R, Siudak Z, Janion-Sadowska A, et al. Effect of day- and night-time admissions on long-term clinical outcomes of patients with acute myocardial infarction treated with percutaneous coronary intervention. Pol Arch Intern Med. 2020; 130(7-8): 570-581, doi: 10.20452/pamw.15398, indexed in Pubmed: 32852908.

5. Park J, Choi KiH, Lee JM, et al. Prognostic implications of doorto-balloon time and onset-to-door time on mortality in patients with ST-segment-elevation myocardial infarction treated with 
primary percutaneous coronary intervention. J Am Heart Assoc. 2019; 8(9): e012188, doi: 10.1161/JAHA.119.012188, indexed in Pubmed: 31041869.

6. Alsamara M, Degheim G, Gholkar G, et al. Is symptom to balloon time a better predictor of outcomes in acute ST-segment elevation myocardial infarction than door to balloon time? Am J Cardiovasc Dis. 2018; 8: 43-47.

7. Chen FC, Lin YR, Kung CT, et al. The Association between Door-to-Balloon Time of Less Than 60 Minutes and Prognosis of Patients Developing ST Segment Elevation Myocardial Infarction and Undergoing Primary Percutaneous Coronary Intervention. Biomed Res Int. 2017; 2017: 1910934, doi: 10.1155/2017/1910934, indexed in Pubmed: 28473978.

8. Halkin A, Singh M, Nikolsky E, et al. Prediction of mortality after primary percutaneous coronary intervention for acute myocardial infarction: the CADILLAC risk score. J Am Coll Cardiol. 2005; 45(9): 1397-1405, doi: 10.1016/j.jacc.2005.01.041, indexed in Pubmed: 15862409.

9. Chan MY, Sun JL, Newby LK, et al. Long-term mortality of patients undergoing cardiac catheterization for ST-elevation and non-ST-elevation myocardial infarction. Circulation. 2009; 119(24): 3110-3117, doi: 10.1161/CIRCULATIONAHA.108.799981, indexed in Pubmed: 19506116.

10. De Luca G, Suryapranata H, van ,t Hof AWJ, et al. Prognostic assessment of patients with acute myocardial infarction treated with primary angioplasty: implications for early discharge. Circulation. 2004; 109(22): 2737-2743, doi: 10.1161/01.CIR.0000131765.73959.87, indexed in Pubmed: 15159293.
11. Karwowski J, Poloński L, Gierlotka M, et al. Post-procedural TIMI flow grade 2 is not associated with improved prognosis in patients with non-ST-segment elevation myocardial infarction undergoing percutaneous coronary revascularization (PLACS registry). Cardiol J. 2016; 23(4): 402-410, doi: 10.5603/ CJ.a2016.0025, indexed in Pubmed: 27296160.

12. Chehab O, Qannus AS, Eldirani M, et al. Predictors of in-hospital mortality in patients admitted with acute myocardial infarction in a developing country. Cardiol Res. 2018; 9(5): 293-299, doi: 10.14740/cr772w, indexed in Pubmed: 30344827.

13. Pocock S, Bueno H, Licour M, et al. Predictors of one-year mortality at hospital discharge after acute coronary syndromes: A new risk score from the EPICOR (long-tErm follow uP of antithrombotic management patterns In acute CORonary syndrome patients) study. Eur Heart J Acute Cardiovasc Care. 2015; 4(6): 509-517, doi: 10.1177/2048872614554198, indexed in Pubmed: 25301783.

14. Garadah TS, Thani KB, Sulibech L, et al. Risk Stratification and in Hospital Morality in Patients Presenting with Acute Coronary Syndrome (ACS) in Bahrain. Open Cardiovasc Med J. 2018; 12: 7-17, doi: 10.2174/1874192401812010007, indexed in Pubmed: 29541260.

15. Quadri G, D’Ascenzo F, Moretti C, et al. Complete or incomplete coronary revascularisation in patients with myocardial infarction and multivessel disease: a propensity score analysis from the „real-life” BleeMACS (Bleeding complications in a Multicenter registry of patients discharged with diagnosis of Acute Coronary Syndrome) registry. EuroIntervention. 2017; 13(4): 407-414, doi: 10.4244/EIJ-D-16-00350, indexed in Pubmed: 28169217. 